\title{
Análisis de Sensibilidad en Simulaciones Fluido Dinámicas de Aneurismas Cerebrales Incluyendo Interacción Fluido Estructura
}

\author{
Alvaro Valencia $^{(1) \star}$, Hernan Figueroa ${ }^{(1)}$, Rodrigo Rivera ${ }^{(2)}$, Eduardo Bravo ${ }^{(2)}$ \\ (1) Universidad de Chile, Departamento de Ingeniería Mecánica, Casilla 2777 Santiago-Chile. \\ (2) Servicio de Neuroradiología, Instituto de Neurocirugía, Asenjo. José Manuel Infante 553, \\ Santiago-Chile.
}

*autor a quien se debe dirigir la correspondencia.

Recibido Oct. 19, 2009; Aceptado Dic. 22, 2009; Versión Final recibida Ene. 13, 2010

\section{Resumen}

Se presenta un estudio sobre sensibilidad de los resultados de simulación fluido dinámica (CFD) en modelos de aneurismas cerebrales, incluyendo la interacción entre la pared arterial y el fluido (FSI). Con 83 modelos reconstruidos de aneurismas cerebrales se hizo un estudio geométrico y se seleccionó un caso para estudiar la fluido dinámica en detalle. Se varió el número de sifones carotideos usados para la simulación CFD. Se realizaron cinco simulaciones FSI variando el modulo de elasticidad arterial y el espesor arterial, y se reportó el efecto de la hipertensión arterial en los resultados. Las simulaciones FSI mostraron que el efecto más relevante en el incremento del estado de esfuerzos en la aneurisma es la hipertensión arterial, con esfuerzos máximos cercanos a $900 \mathrm{kPa}$. Se concluye que las simulaciones FSI se deben usar para determinar simultáneamente el estado de esfuerzos de la pared y la fluido dinámica dentro de aneurismas.

Palabras clave: simulación fluido dinámica, aneurisma cerebral, hipertensión, CFD,FSI

\section{Sensibility Analysis in Computational Fluid Dynamics of Cerebral Aneurysm including Fluid Structure Interaction}

\begin{abstract}
A sensitivity study of the results provided by computational fluid dynamics (CFD) in models of cerebral aneurysms including fluid structure interaction (FSI) was performed. With 83 reconstructed models of cerebral aneurysms, a geometrical study was done and one case was selected for a detailed study of the computational fluid dynamics. In this model the number of carotid siphon used for the CFD simulations was varied. In the FSI study, five simulations were conducted varying the elastic modulus of the artery and the arterial wall thickness, and the effect of hypertension on the results was reported. The FSI simulations have shown that the most important parameter that affects the aneurysm wall stress state is the arterial hypertension with maximum stress of around $900 \mathrm{kPa}$. It is concluded that FSI simulations must be used to simultaneously determine the wall stress and the fluid dynamics inside cerebral aneurysm.
\end{abstract}

Keywords: computational fluid dynamics, cerebral aneurysm, hypertension, CFD, FSI 


\section{INTRODUCCIÓN}

Una aneurisma cerebral es una dilatación patológica de una arteria que generalmente se desarrolla en las regiones anteriores y posteriores del círculo de Willis en la base del cerebro. Las aneurismas se clasifican en tipo fusiformes o tipo saco. La mayoría de las aneurismas cerebrales son tipo saco y se ubican en una posición lateral de una arteria o en bifurcaciones arteriales. La ruptura de una aneurisma provoca una hemorragia con graves consecuencias para el paciente, Schievink (1997). Los tratamientos clínicos actuales de esta enfermedad son la inserción de coils y stents por medio endovascular y el clipado quirúrgico.

Las aneurismas son estructuras de pared muy delgada y la ruptura ocurre principalmente en la región del domo de la aneurisma y puede ser explicado en parte por el debilitamiento de las propiedades mecánicas de la pared en esa región (Humphrey y Canham, 2000). La resistencia mecánica de pared de la aneurisma se ha estimado que varía entre 0.73 MPa y 1.9 MPa (MacDonald et al., 2000).

La importancia de la fluido dinámica se debe a que el flujo sanguíneo controla el esfuerzo de corte en la pared de la aneurisma (WSS) y como el WSS es distinto que en una pared arterial sana se produce un ciclo de apoptosis celular afectando localmente las propiedades mecánicas de la pared aneurisma produciendo crecimiento y posterior ruptura de esta, (Frösen et al., 2004; Pentimalli et al., 2004).

Usando CFD, se han realizado una serie de estudios fluido dinámicos de aneurismas cerebrales. Shojima et al. 2004 presentan una correlación entre WSS y geometría de la aneurisma. Cebral et al. (2005) han caracterizado la fluido dinámica en las aneurismas cerebrales y Valencia et al. (2008) han presentado correlaciones para WSS en función de parámetros geométricos de la aneurisma. El objetivo de este estudio es reportar el efecto del número de sifones carotideos de arteria antes de la aneurisma. Reportar los efectos del modulo de elasticidad y espesor arterial en el WSS, esfuerzos y deformaciones de la pared de la aneurisma y estudiar el efecto de la hipertensión arterial.

\section{MODELOS MATEMATICOS}

Las geometrías de los modelos de aneurismas cerebrales fueron obtenidas de un equipo de angiografía digital 3D modelo Phillips Integris Allura, que entrega una imagen en formato VRML (Lenguaje para Modelado de Realidad Virtual). Con metodología propia desarrollada en nuestro proyecto de obtiene una geometría en formato CAD (Diseño Asistido por Computador) simplificada de la real. La metodología se describió en detalle en Valencia at al. (2008). La geometría se escala usando la imagen médica, que contiene tres medidas principales: alto, diámetro en el centro y tamaño del cuello de la aneurisma. Posteriormente se importa el archivo en formato Parasolid en el programa de elementos finitos ADINA, donde se definen los modelos matemáticos del fluido y sólido a usar, además de las condiciones de borde adecuadas al problema. En los casos CFD (Mecánica de Fluidos Computacional) se resuelve solo el modelo de fluido y el sólido es la condición de borde. Para los problemas FSI (Interacción de Fluido con Estructura) se resuelve simultáneamente el modelo fluido acoplado con el modelo de sólido elegido.

Los criterios de selección de casos y de aproximación de las geometrías esta basado en la experiencia médica clínica de nuestro grupo. El caso de estudio en este trabajo es una aneurisma tipo saco localizada en el segmento carotideo y se muestra en la Fig. 1. El volumen total es de 1005 $\mathrm{mm}^{3}$. La geometría incluye tres sifones carotideos antes de la aneurisma cerebral.

\section{Modelo de Fluido}

El flujo está descrito por las ecuaciones de continuidad y de Navier-Stokes para un fluido incompresible y en flujo laminar:

$$
\begin{aligned}
& \nabla \cdot \mathbf{u}=0 \\
& \rho_{f}\left(\partial \mathbf{u} / \partial t+\left(\left(\mathbf{u}-\mathbf{u}_{\mathbf{g}}\right) \cdot \nabla\right) \mathbf{u}\right)=-\nabla p+\nabla \cdot \boldsymbol{\tau}
\end{aligned}
$$




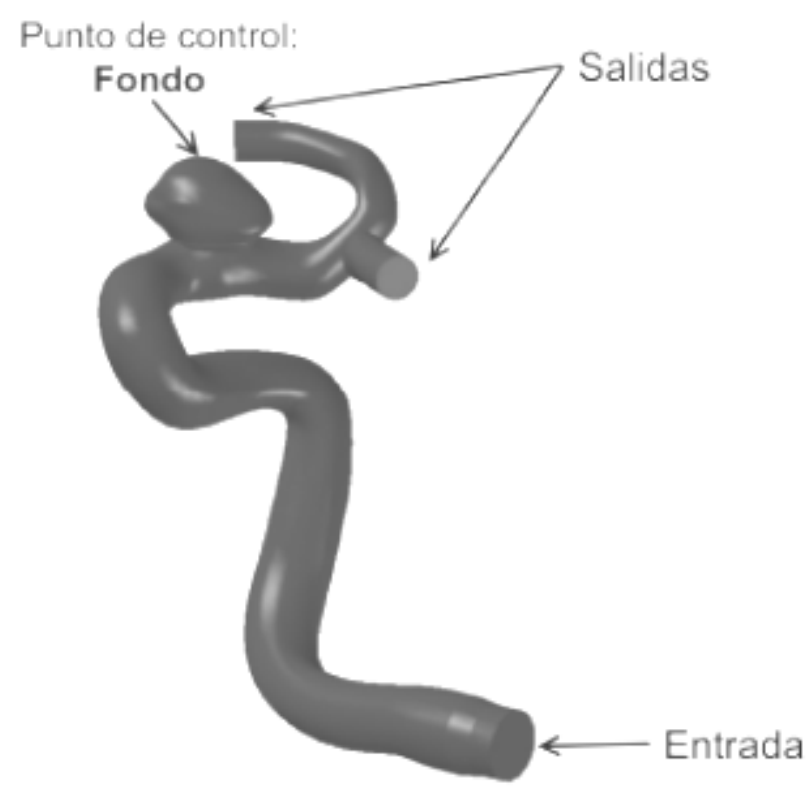

Fig. 1: Modelo CAD de aneurisma cerebral real.

Donde $u_{g}$ es la velocidad de movimiento de la coordenada en los problemas tipo FSI. La sangre se considera un fluido no Newtoniano y se usa el modelo de Carreau de tres parámetros (Cho y Kensey, 1991), para describir la variación de la viscosidad con la tasa de deformación:

$$
\mu=\mu_{\infty}+\left(\mu_{0}-\mu_{\infty}\right) \cdot\left(1+\mathrm{k} \dot{\gamma}^{2}\right)^{\mathrm{m}}
$$

Donde $\mu_{\infty}$ es la viscosidad a alta velocidad de corte, $\mu_{0}$ es la viscosidad sin deformación y $\gamma$ es la velocidad de corte. Los parámetros $\mathrm{k}$ y $\mathrm{m}$ controlan el tamaño de la zona de transición del fluido. En este trabajo hemos considerado: $\mu_{\infty}=0.00345 \mathrm{Ns} / \mathrm{m}^{2}, \mu_{0}=0.056 \mathrm{Ns} / \mathrm{m}^{2}, k=10.976$ y $\mathrm{m}=-0.3216$ (Johnston et al., 2004).

\section{Modelo de sólido}

El movimiento del sólido está regido por la conservación de movimiento:

$$
\nabla \cdot \sigma_{\mathrm{S}}=\rho_{\mathrm{S}} \dot{\mathbf{u}}_{\mathrm{g}}
$$

Donde $\sigma_{\mathrm{s}}$ es el esfuerzo del sólido y $\mathrm{u}_{\mathrm{g}}$ es la velocidad de la coordenada. El espesor de la pared arterial de una aneurisma cerebral es de alrededor de $0.086 \mathrm{~mm}$, el espesor de la pared arterial normal se considera de $0.4 \mathrm{~mm}$, para una arteria típica de diámetro $4.8 \mathrm{~mm}$. Estos pequeños valores permiten asumir la pared arterial con un modelo tipo Shell (Bathe, 1996).

El material que compone una aneurisma tiene un comportamiento hiperelástico y anisotrópico. El comportamiento del material es descrito por una función de energía de deformación w, tal que:

$$
\sigma=\partial \mathrm{w} / \partial \lambda
$$

Usando los datos experimentales de Seshaiyer at al. (2001), hemos aproximado el comportamiento de la curva esfuerzo $\sigma$ versus la deformación $\lambda$ en un test biaxial, a un modelo del tipo Mooney-Rivlin hiperelástico, y se obtiene que: 


$$
\sigma=\partial \mathrm{w} / \partial \lambda=\mathrm{C} \lambda\left(\mathrm{C}_{1}+\mathrm{C}_{3}\right)\left(\lambda^{2}-1\right) \mathrm{e}^{1 / 4\left(\mathrm{C}_{1}+\mathrm{C}_{2}+2 \mathrm{C}_{3}\right)\left(\lambda^{2}-1\right)^{2}}
$$

Tomando la pendiente de esta curva, se obtiene el módulo de elasticidad del material E=7 MPa. También hemos considerado un modelo hiperalástico propuesto por Delfino et al. (1997), con un pre estiramiento de un $20 \%$. Con el mismo procedimiento se obtiene un modulo de elasticidad E=10 MPa, en este caso.

\section{Condiciones Frontera}

Para las simulaciones CFD y FSI se consideró un perfil de velocidad a la entrada de la arteria promedio, obtenido de exámenes de ultrasonido Doppler realizado a 36 pacientes con aneurisma cerebral. Con el examen de ultrasonido Doppler se determina la variación temporal del flujo sanguíneo en la arteria carótida del paciente. El examen utiliza ultrasonografía dúplex para visualizar el flujo sanguíneo. El perfil obtenido se muestra en la Fig. 2a. La variación espacial a la entrada de este perfil se implemento usando el perfil obtenido por Womersley (1955). Es la solución analítica al problema de un flujo pulsante en un tubo y descrito en detalle también en Zamir (2000). A la salida de las arterias se impuso un pulso de presión normal y en una caso hipertenso, mostrados en la Fig. 2b. Este pulso se obtuvo tomando valores estándar de presión arterial. La presión exterior a las arterias se consideró constante y se le asignó un valor de referencia de $400 \mathrm{~Pa}$, producido por el líquido cerebral, (Humphrey y Canham, 2000). Por ser este valor muy pequeño con la presión al interior de la arteria, una variación de este no afecta los resultados.
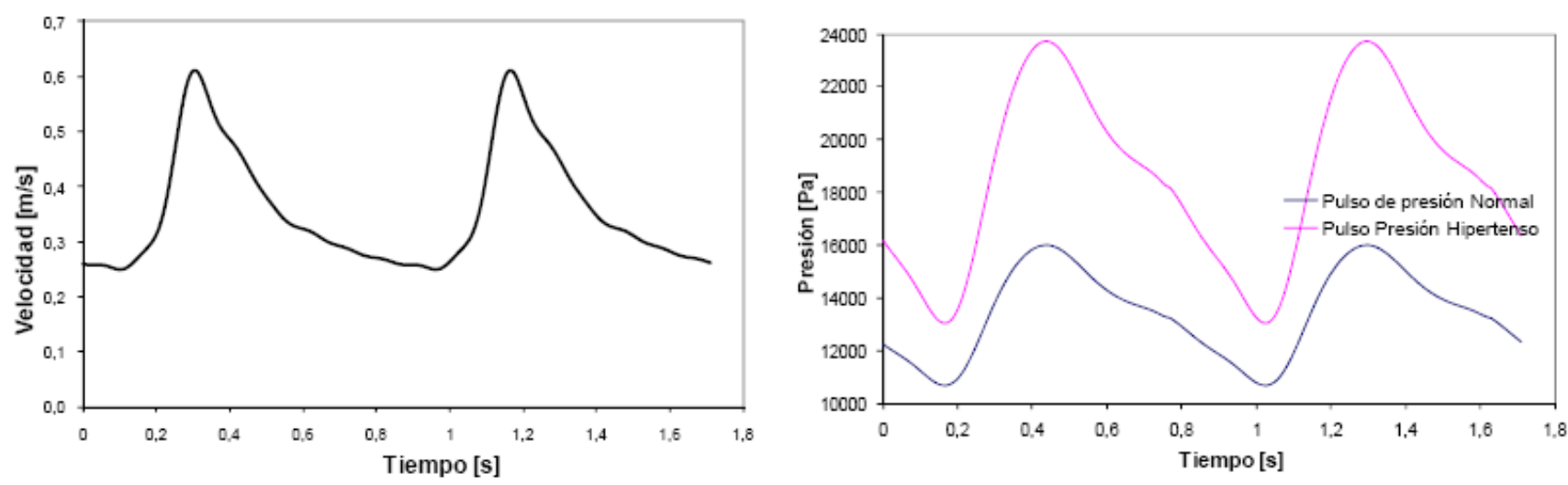

Fig. 2: a) Variación temporal de la velocidad promedio a la entrada del modelo, b) Variación temporal de la presión en las salidas del modelo.

\section{METODO NUMERICO}

El modelo de fluido y de sólido se resolvieron usando los módulos CFD y FSI del programa de elementos finitos ADINA v8.5. En la parte de fluido se uso el módulo de flujo laminar con fluido no Newtoniano, usando el modelo de Carreau. El perfil de velocidad de entrada se entrega mediante una subrutina construida en Matlab ad hoc, indicando la velocidad en cada nodo de la sección de entrada al dominio de solución numérica. Para el cálculo se consideró interpolación temporal y espacial de primer orden y se usó un criterio de convergencia entre cada paso de tiempo de 0.001 para las ecuaciones de continuidad y de movimiento en el fluido. En el sólido se considera toda la geometría con las mismas propiedades elásticas (arteria y aneurisma), se considera empotramiento en la entrada y en la salida de las arterias. El sistema completa incluyendo la arteria se puede deformar.

Se usaron elementos con interpolación basada en la condición del flujo (FCBI) en el fluido, ver manual ADINA, y un método iterativo para el acoplamiento directo con el sólido en los casos FSI. Las mallas usadas fueron de 390,000 elementos en el fluido, en el caso con dos sifones carotideos 
precedentes a la aneurisma y 53,000 elementos en el sólido considerado como Shell. El paso de tiempo fue de $0.01 \mathrm{~s}$ y se consideraron 2 ciclos cardiacos consecutivos de $0.86 \mathrm{~s}$.

Para definir el tamaño de la malla en el fluido se usó el criterio de usar más de 500 elementos $/ \mathrm{mm}^{3}$, definidos como adecuados para simulaciones CFD y FSI en aneurismas cerebrales usando la misma metodología y software por Valencia y Ledermann (2008). Para un volumen de fluido de $722.87 \mathrm{~mm}^{3}$ en el caso con dos sifones carotideos, se usaron 388,472 elementos, que corresponde a una densidad de 538 elementos $/ \mathrm{mm}^{3}$. Esta densidad de elementos se mantuvo, para los casos CFD con uno y tres sifones y en los casos con simulaciones FSI.

Usando el mismo programa ADINA se obtienen los campos de velocidad y presión en el fluido y los campos de esfuerzo y deformación en el sólido.

\section{RESULTADOS Y DISCUSIÓN}

Se define el factor geométrico NSI (índice de no esfericidad) de una aneurisma como:

$$
N S I=1-(18 \pi)^{1 / 3} \frac{V^{2 / 3}}{S}
$$

EI NSI da cuenta de la diferencia adimensional de la forma de una aneurisma con respecto a una semiesfera (Raghavan et al., 2005). La Fig. 3 muestra el resultado de aplicar este factor a nuestras 83 aneurismas reconstruidas en este proyecto, son 41 casos con ruptura previa y 42 casos no rotos. La figura muestra la distribución de frecuencia para el NSI. El valor NSI=0.153 indica el punto donde la frecuencia de casos no rotos iguala a 1 -frecuencia de los casos rotos. Con esto se obtiene el importante resultado de que si $\mathrm{NSI}<0.15$ el $70 \%$ de los casos no presenta ruptura de aneurisma. Raghavan (2005) en su estudio de 23 casos, encontró una diferencia estadística significativa del valor promedio del NSI en aneurismas sin ruptura previa (0.156), comparado con el NSI promedio de aneurismas que habían sufrido ruptura previa (0.233). Esto indica de que el factor geométrico NSI podría ser un buen indicador para relacionar geometría de aneurismas cerebrales con su estado de esfuerzos y deformación.

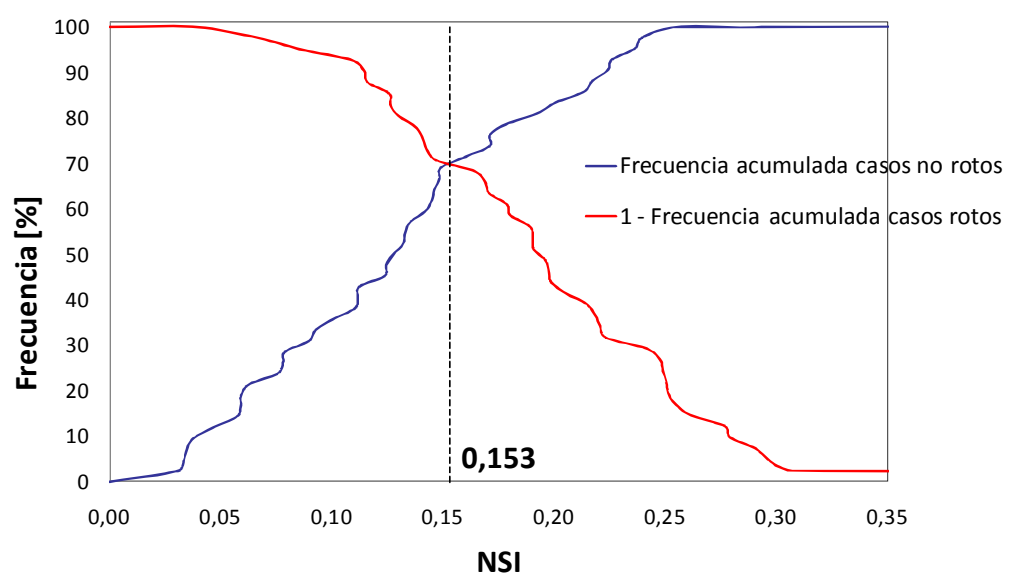

Fig. 3. Distribución de frecuencias de casos en función de NSI para 83 aneurismas.

La Fig. 4 muestra la geometría de los 3 casos considerados para las simulaciones CFD, el objetivo es determinar el efecto del número de sifones previos a la aneurisma en la fluido dinámica de ésta. La Fig. 5 muestra la distribución de la magnitud de la velocidad antes de la aneurisma para los tres casos, se observa que los resultados usando dos tres sifones carotideos previos a la aneurisma entregan los mismos resultados. La distribución de velocidades muestra una zona de alta velocidad inducida por la forma del sifón carotideo, alcanzando $1.3 \mathrm{~m} / \mathrm{s}$. La forma del perfil de velocidad a la 
salida del sifón determina el flujo de entrada a la aneurisma. La Tabla 1 muestra una comparación cuantitativa con respecto a los resultados fluido dinámicos usando 3 sifones carotideos. La diferencia en WSS, velocidad y presión son pequeñas usando 2 sifones. Se concluye que 2 sifones previos son suficientes y con esa configuración se realizan las simulaciones FSI.

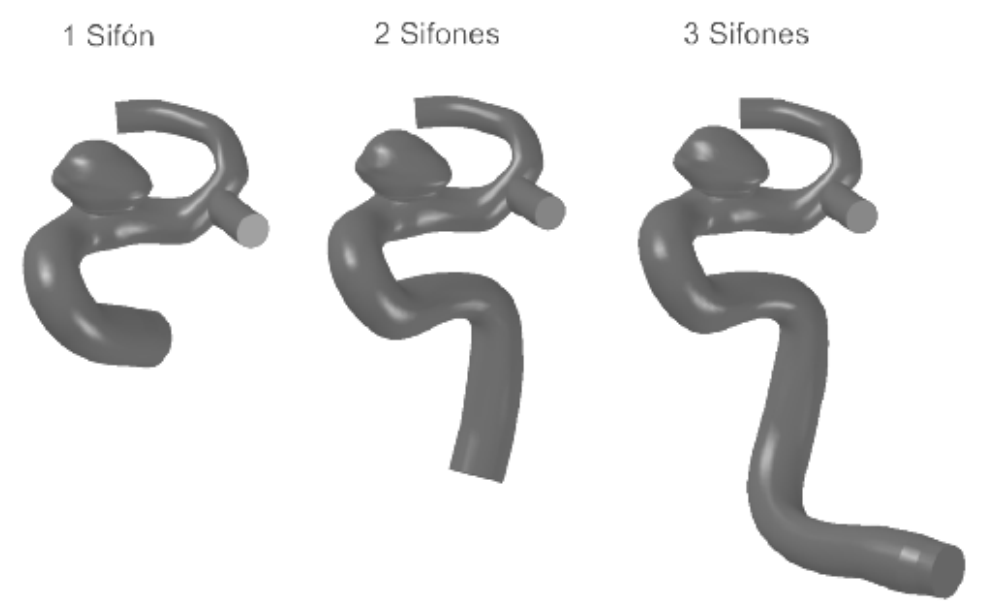

Fig. 4. Geometrías usadas para CFD.

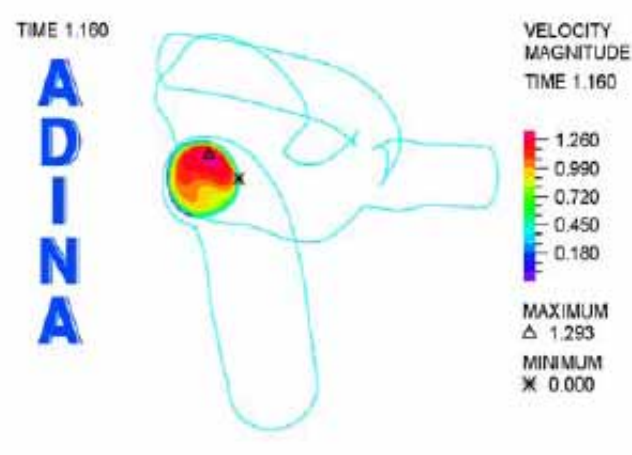

1 Sifón

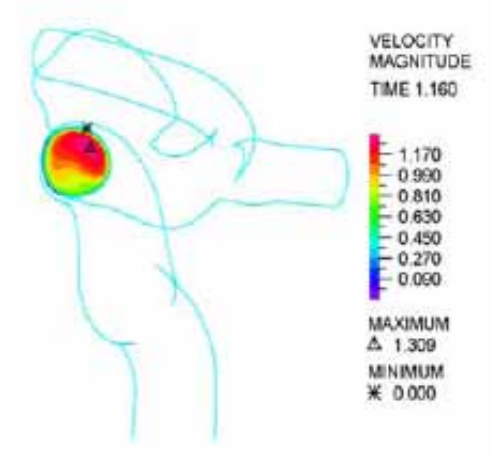

2 Sifones

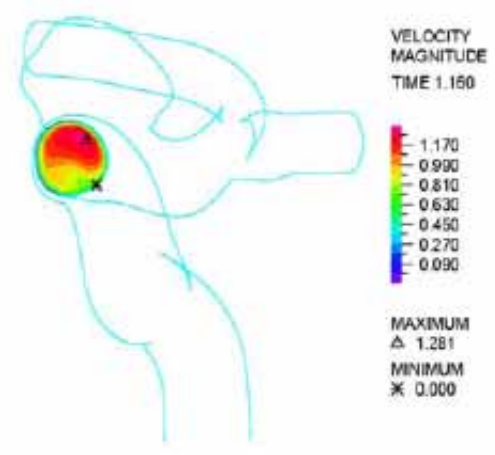

3 Sifones

Fig. 5 Distribución de magnitud de velocidad en la sístole.

Tabla 1. Resultados CFD de WSS en el fondo de la aneurisma y velocidad máxima en el plano de entrada a la aneurisma, para los casos con 3 ,2 y 1 sifón respectivamente. Las diferencias porcentuales se calculan con respecto del caso con 3 sifones.

\begin{tabular}{|c|c|c|c|c|c|}
\hline $\begin{array}{c}\text { WSS fondo } \\
{[\mathrm{Pa}]}\end{array}$ & $\begin{array}{c}\text { Diferencia } \\
\%\end{array}$ & $\begin{array}{c}\text { Velocidad máxima } \\
\text { plano entrada }[\mathrm{m} / \mathrm{s}]\end{array}$ & $\begin{array}{c}\text { Diferencia } \\
\%\end{array}$ & $\begin{array}{c}\text { Caída presión } \\
\text { máxima }[\mathrm{KPa}]\end{array}$ & $\begin{array}{c}\text { Diferencia } \\
\%\end{array}$ \\
\hline 9.102 & 0 & 0.8285 & 0 & 5.373 & 0 \\
\hline 9.302 & 2.2 & 0.8460 & 2.4 & 5.060 & 5.8 \\
\hline 8.823 & 3.1 & 0.8675 & 4.7 & 4.632 & 13.8 \\
\hline
\end{tabular}

La Fig. 6 muestra la distribución de presión obtenida con CFD y se aprecia claramente de que la presión es uniforme alrededor de la aneurisma para el caso con 2 sifones carotideos con un valor de $18.7 \mathrm{kPa}$ en la sístole.

La Fig. 7 muestra la distribución de velocidad en tres planos en la aneurisma, se observan estructuras vorticiales al interior de la aneurisma, que provocan que el esfuerzo de corte en la pared 
(WSS) varíe significativamente en la superficie de la aneurisma, ver Fig. 8. En el fondo de la aneurisma el WSS alcanza solo $8 \mathrm{~Pa}$ en la sístole. La distribución del WSS en la geometría completa y el detalle del WSS en la aneurisma obtenidas con simulación FSI se muestran en la Fig. 9. Se observa que los valores de WSS en la arteria son mucho mas grandes que en la aneurisma, esto tiene gran importancia porque podría confirmar que debido a pequeños valores del WSS en la aneurisma las células de endotelio de la pared podrían iniciar un ciclo de apoptosis celular con la consecuente degradación de las propiedades estructurales de la pared.

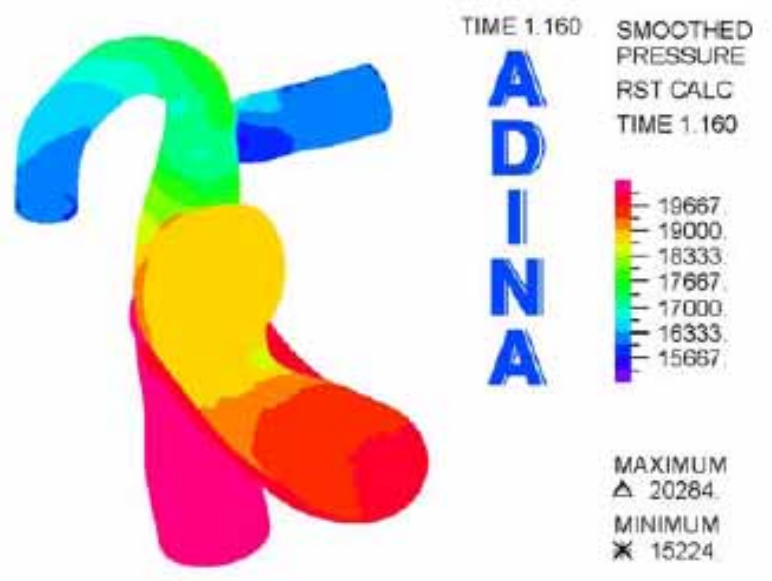

Fig. 6. Distribución de presión en la sístole, simulación CFD.

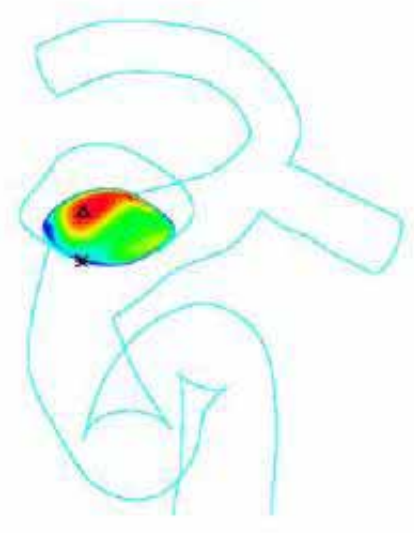

PLANO ENTRADA

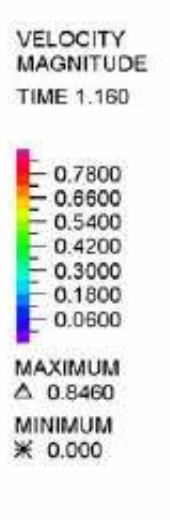

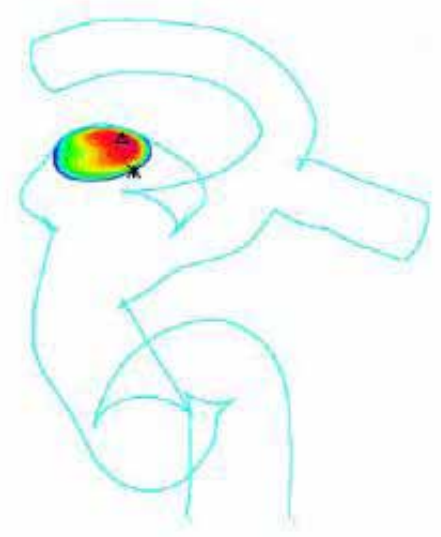

PLANO SUPERIOR

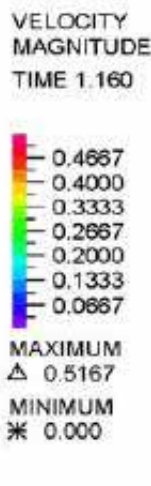

VELOCITY

MAGNITUDE

TIME 1.160

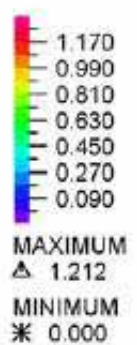

PLANO TRANSVERSAL

Fig. 7. Distribución de velocidad en tres planos de la aneurisma, simulación CFD. 


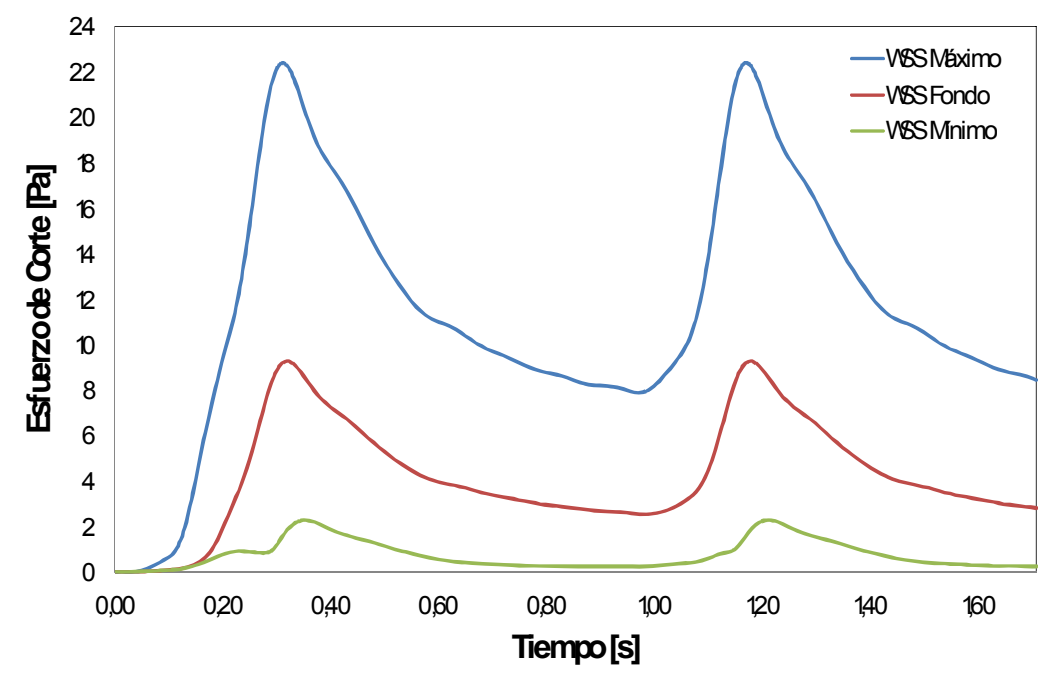

Fig. 8. WSS máximo, mínimo y en el fondo de la aneurisma en función del tiempo, simulación CFD.
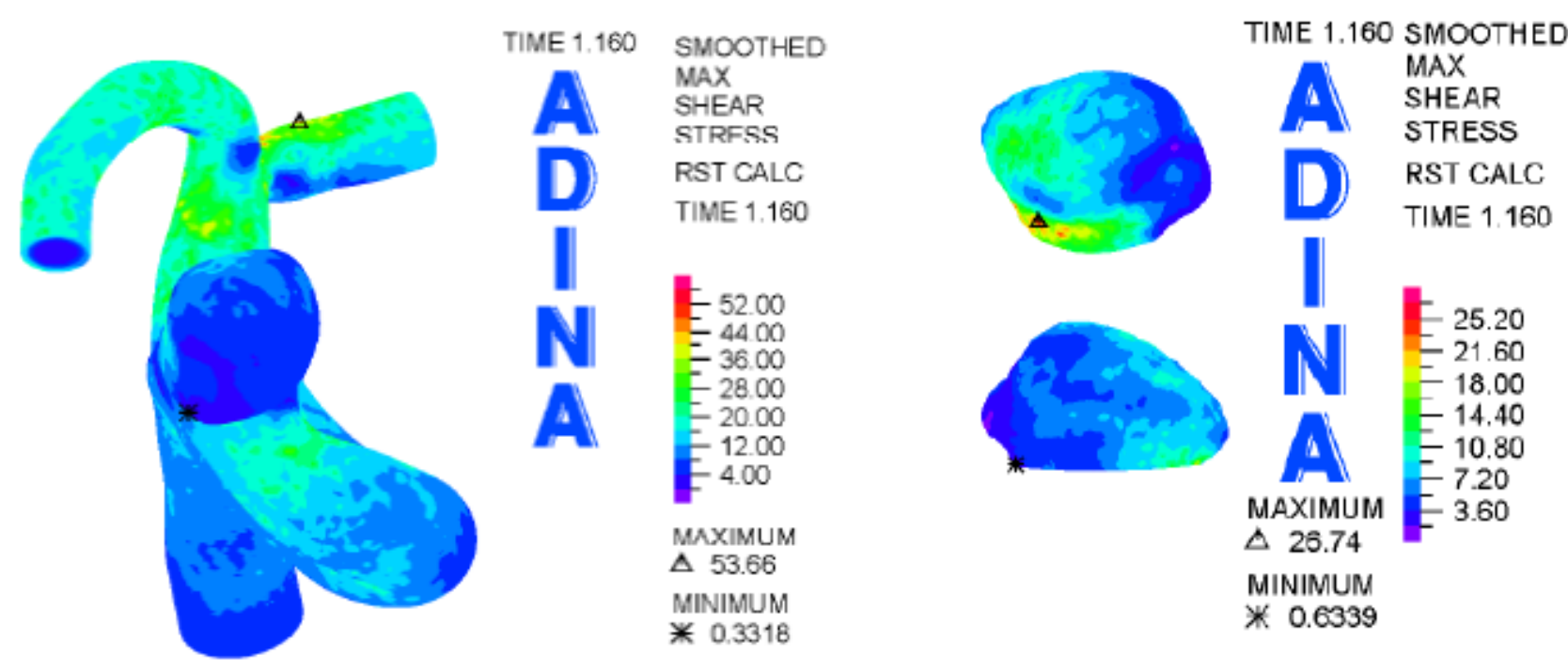

Fig. 9 a) Distribución de WSS en toda la geometría, b) distribución de WSS en la aneurisma, obtenidas en la sístole con simulación FSI.

La distribución de esfuerzos efectivos y deformación principal obtenidos con la simulación FSI se muestran en la Fig. 10. Se consideró el caso de pulso normal, modelo de pared variable (espesor pared arteria $0.4 \mathrm{~mm}$, espesor pared aneurisma $0.086 \mathrm{~mm}$ ) y modulo de elasticidad $\mathrm{E}=7 \mathrm{MPa}$. Este caso se considera el caso base para las variaciones que se reportan mas adelante. Debido a que el espesor de la arteria en este modelo es mucho mayor que el espesor de la aneurisma, los esfuerzos y las deformaciones en la pared arterial son muy pequeñas.

Se realizó un estudio del efecto de la hipertensión arterial en la simulación FSI, dejando todas las otras variables sin modificar. Se mantuvieron constantes los espesores de la pared arterial y de la aneurisma, así como el módulo de elasticidad del modelo completo. La Fig. 11a muestra que la hipertensión arterial aumenta en forma significativa los esfuerzos en la pared de la aneurisma alcanzando los $900 \mathrm{kPa}$ en la sístole. Este valor está en intervalo del valor de ruptura reportado por MacDonald et al. (2000), para aneurismas cerebrales y por lo tanto se tiene una situación que puede ser crítica. 

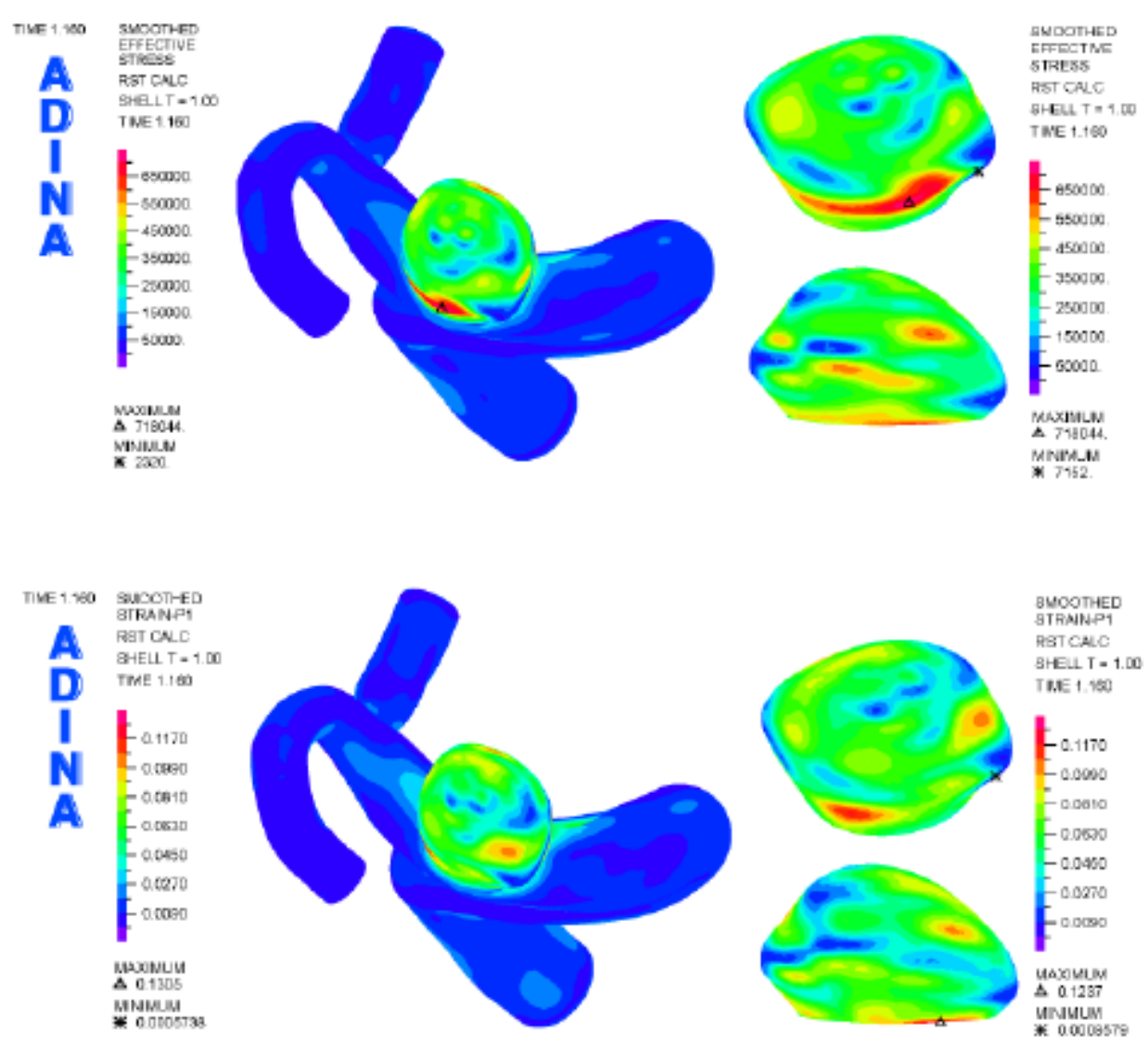

Fig. 10 a) Distribución de esfuerzo efectivo, b) distribución de deformación principal 1, obtenidas en la sístole con simulación FSI normal.
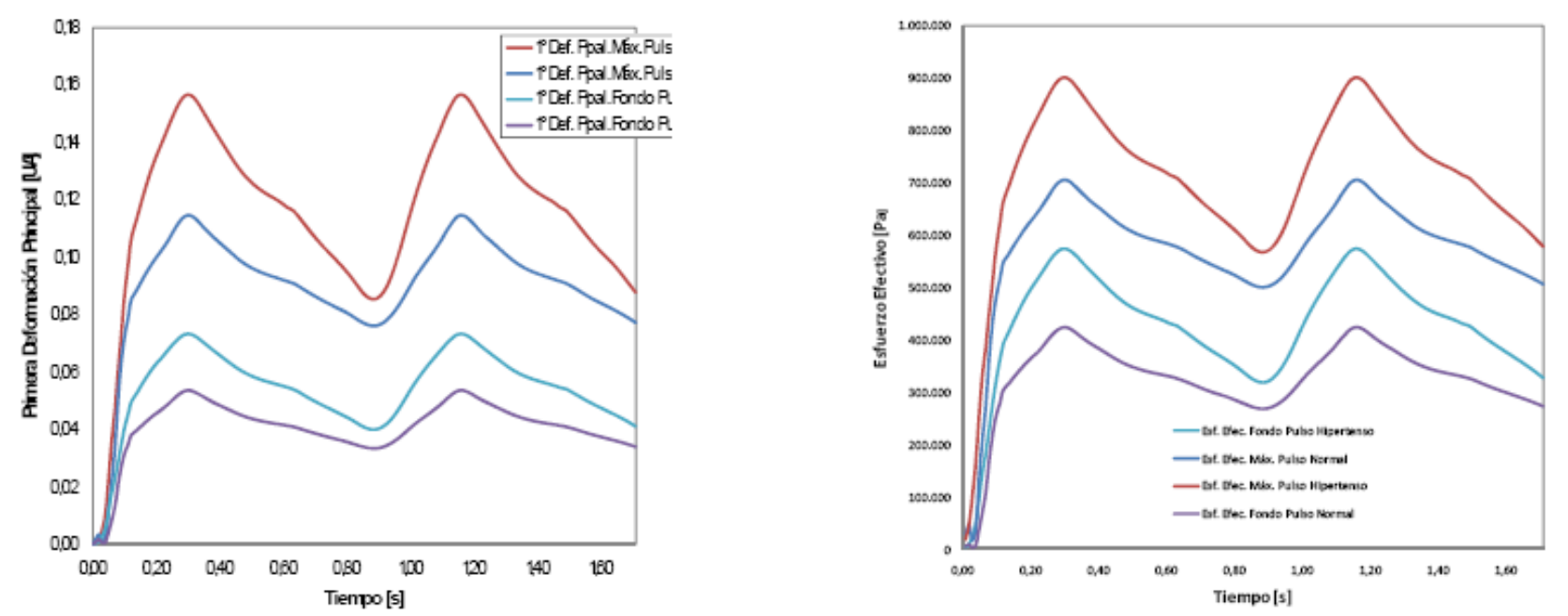

Fig. 11 a) Variación temporal del esfuerzo efectivo, b) variación temporal de la primera deformación principal, en dos puntos de la aneurisma para caso normal e hipertenso.

La Fig. 11b muestra la variación de la primera deformación principal en el fondo de la aneurisma y el valor máximo con respecto al tiempo tanto para el caso normal como para el caso hipertenso. Con hipertensión la deformación máxima es cerca de 0.16 en la sístole y aumenta en $35 \%$ con respecto del caso con presión arterial normal. Este es un incremento significativo de la deformación de la pared de la aneurisma.

Finalmente, se realizaron estudios FSI para el caso con presión normal, variando el espesor de la pared, considerando en un caso un modelo con pared de espesor de $0.086 \mathrm{~mm}$ y el otro con espesor 
constante pero de $0.4 \mathrm{~mm}$ (todo el modelo con espesor de arteria) y se corrió un caso con un modulo de elasticidad $\mathrm{E}=10 \mathrm{MPa}$ (modelo Delfino equivalente). La Fig. 12 muestra la distribución del esfuerzo efectivo en la sístole, para los casos simulados con modelos de pared uniforme, de $0.4 \mathrm{~mm}$ y $0.086 \mathrm{~mm}$, o de diámetro exterior de $4.94 \mathrm{~mm}$ y $4.312 \mathrm{~mm}$, respectivamente.

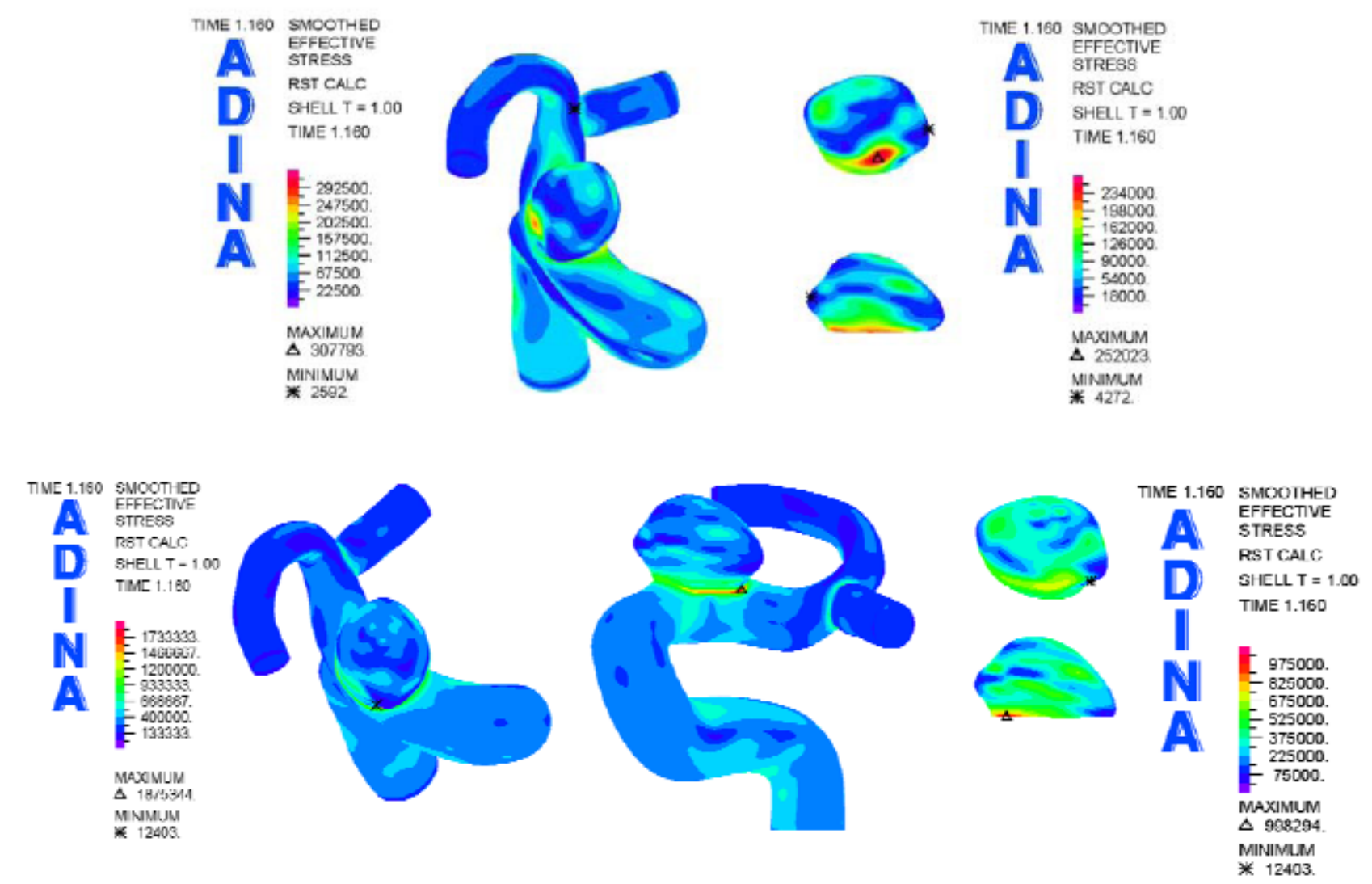

Fig. 12 a) Distribución de esfuerzo efectivo modelo de pared uniforme $0.4 \mathrm{~mm}$, b) distribución de esfuerzo efectivo modelo de pared uniforme $0.086 \mathrm{~mm}$. Obtenidos en la sístole con FSI.

En el modelo con espesor de arteria se obtienen valores del esfuerzo menor a $260 \mathrm{kPa}$ en la aneurisma, considerados no realistas. Esto muestra la importancia del espesor de los modelos de pared en el estudio de la mecánica de sólidos en aneurismas cerebrales. La predicción de esfuerzos mejora notablemente si se considera el espesor de la aneurisma en el modelo completo.

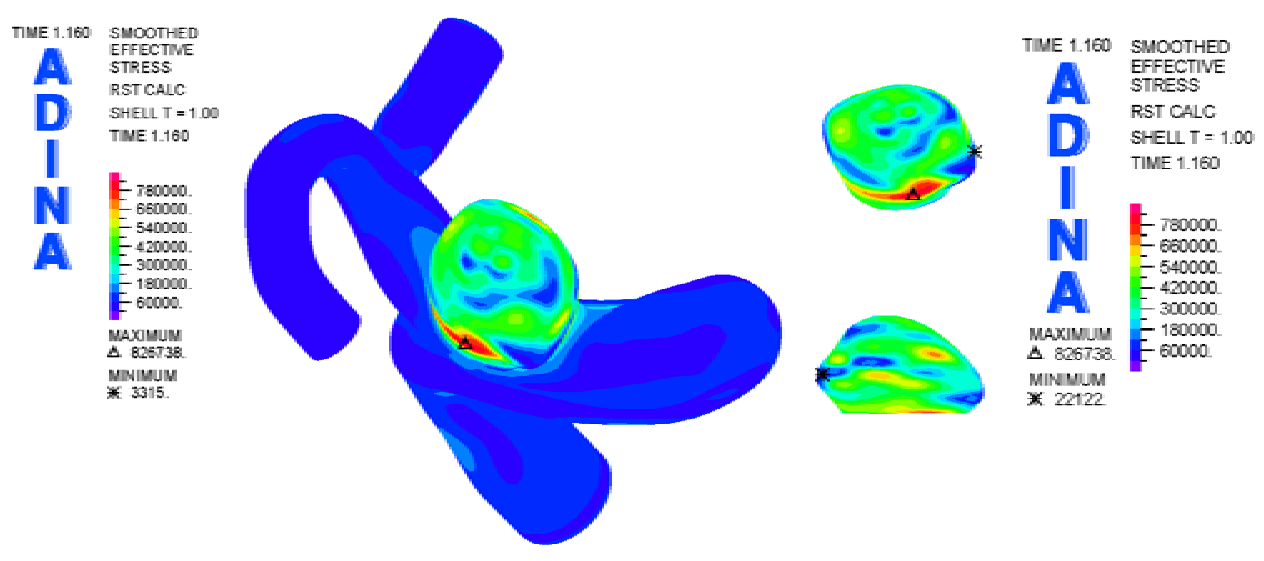

Fig. 13. Distribución de esfuerzo efectivo en la sístole obtenida con simulación FSI, E=10 MPa.

La Fig. 13 muestra el efecto de aumentar el modulo de elasticidad en los esfuerzos efectivos. Un $30 \%$ de aumento del módulo de elasticidad significa un incremento en el esfuerzo máximo de solo un 
$15 \%$ en el modelo de pared variable. Finalmente, la Fig. 14 muestra la variación del WSS con los distintos modelos en el fondo de la aneurisma y el WSS máximo en la aneurisma. El modelo con espesor arteria se aleja del modelo de pared variable.

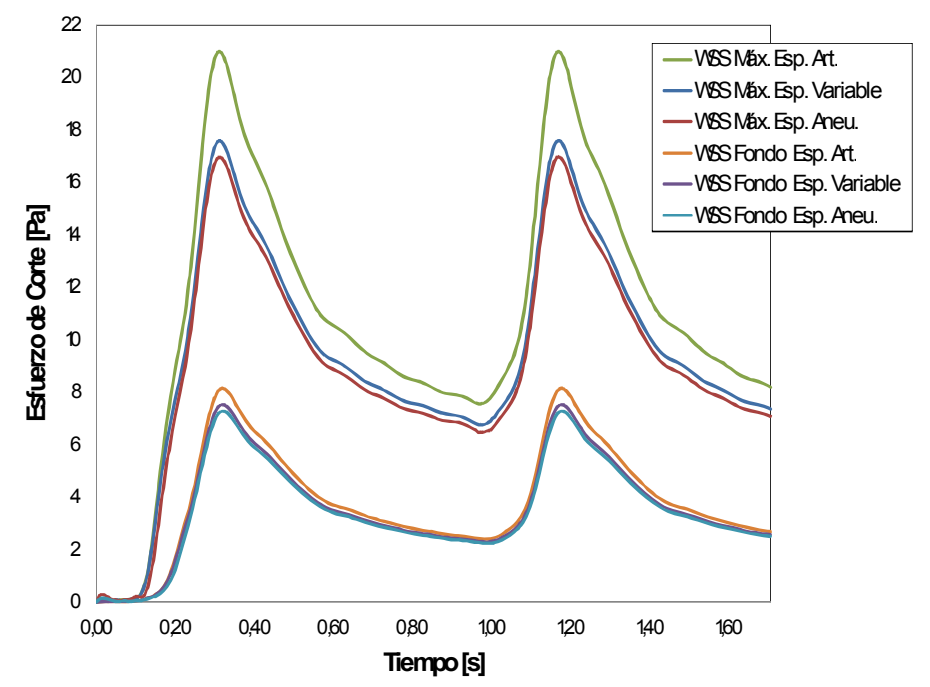

Fig. 14. Variación de WSS con distintos modelos de espesor de pared en simulaciones FSI.

No se conocen resultados experimentales o reales de la fluido dinámica en aneurismas cerebrales en pacientes humanos en vivo, debido a su ubicación en general en la base del cerebro, para comparar los resultados de este estudio. Resultados de simulaciones CFD realizadas recientemente (Rayz et al. 2008; Torii et al. 2009; Jiang et al. 2009; Baek et al. 2009 y Sforza et al. 2009) permiten comparar los órdenes de magnitud del WSS y de la presión en la aneurisma, encontrándose una buena concordancia.

\section{CONCLUSIONES}

En este trabajo se reporta una distribución de frecuencias de casos sin ruptura previa de aneurismas cerebrales usando nuestra base de datos de 83 casos reconstruidos. Se encontró que el valor del parámetro geométrico adimensional $\mathrm{NSI}=0.15$ es un valor interesante de considerar para ayudar a determinar la probabilidad de ruptura.

Simulaciones CFD demostraron que el uso de dos sifones carotideos antes de la aneurisma era suficiente para reproducir la fluido dinámica en la aneurisma. Las simulaciones FSI mostraron que el efecto más relevante en el incremento del estado de esfuerzos en la aneurisma es la hipertensión arterial. Se encontraron esfuerzos máximos cerca de $900 \mathrm{kPa}$.

\section{AGRADECIMIENTOS}

El financiamiento de esta investigación se realizó a través del proyecto FONDECYT № 1070773.

\section{REFERENCIAS}

ADINA, Theory and Modeling Guide. Volume I, III. ADINA R \& D, Inc. Watertown, MA, USA, (2004).

H. Baek, M. Jayaraman y G. Karniadakis, Wall shear stress and pressure distribution on aneurysms and infundibulae in the posterior communicating artery bifurcation. Annals of Biomedical Engineering, 12, 2469-2487 (2009).

K.J. Bathe, Finite Element Procedures, Prentice Hall, New Jersey, (1996).

J. Cebral y otros 5 autores, Characterization of cerebral aneurysms for assessing risk of rupture by using patient-specific computational hemodynamics models. American Journal of Neuroradiology, 26, 2550-2559 (2005). 
Y. Cho y K. Kensey, Effects of the non-Newtonian viscosity of blood on flows in a diseased arterial vessel. Part I: steady flows. Biorheology, 28, 241-262 (1991).

A. Delfino y otros 4 autores, Residual strain effects on the stress field in a thick wall finite element model of the human carotid bifurcation. Journal of Biomechanics, 30, 777-786 (1997).

J. Frösen y otros 6 autores, Remodeling of saccular cerebral artery aneurysm wall is associated with rupture-Histological analysis of 24 unruptured and 42 ruptured cases, Stroke, 35, 2287-2293 (2004).

J. Humphrey y P. Canham, Structure, mechanical properties, and mechanics of intracranial saccular aneurysms. Journal of Elasticity, 61, 49-81 (2000).

J. Jiang y Ch. Strother, Computational fluid dynamics simulations of intracranial aneurysms at varying heart rates: a "patient-specific" study, Journal of Biomechanical Engineering 131, 091001-1, (2009).

B. Johnston y otros 3 autores, Non-Newtonian blood flow in human right coronary arteries: steady state simulations. Journal of Biomechanics, 37, pp. 709-720 (2004).

D. MacDonald, H. Finlay y P. Canham, Directional wall strength in saccular brain aneurysms from polarized light microscopy. Annals of Biomedical Engineering, 28, 533-542 (2000).

L. Pentimalli y otros 10 autores, Role of apoptosis in intracranial aneurysm rupture. Journal of Neurosurgery, 101, 1018-1025 (2004).

M. Raghavan, B. Ma y R. Harbaugh, Quantified aneurysm shape and rupture risk. Journal of Neurosurgery 102, 355-362 (2005).

V. Rayz y otros 7 autores, Numerical modeling of the flow in intracranial aneurysms: prediction of regions prone to thrombus formation. Annals of Biomedical Engineering, 36, 1793-1804 (2008).

W. Schievink, Intracranial Aneurysms. The New England Journal of Medicine, 336, 28-40 (1997).

P. Seshaiyer y otros 4 autores, Multiaxial mechanical behavior of human saccular aneurysms. Computer Methods in Biomechanics and Biomedical Engineering, 4, 281-289 (2001).

M. Shojima y otros 7 autores, Magnitude and role of wall shear stress on cerebral aneurysm computational fluid dynamic study of 20 middle cerebral artery aneurysms. Stroke, 35, 2500-2505 (2004).

D. Sforza, Ch. Putman y J. Cebral, Hemodynamics of cerebral aneurysms. Annual Review of Fluid Mechanics, 41, 91-107 2009.

R. Torii y otros 4 autores, Fluid-structure interaction modeling of blood flow and cerebral aneurysm: significance of artery and aneurysm shapes. Computer Methods in Applied Mechanics and Engineering, 198, 3613-3621 (2009).

A. Valencia y otros 4 autores, Blood flow dynamics and fluid-structure interaction in patient-specific bifurcating cerebral aneurysms. Int. J. for Numerical Methods in Fluids, 58, 1081-1100 (2008).

A. Valencia y otros 4 autores, Blood flow dynamics in patient-specific cerebral aneurysm models: The relationship between wall shear stress and aneurysm area index. Medical Engineering \& Physics, 30, 329340 (2008).

J.R. Womersley, Oscillatory motion of a viscous liquid in a thin-walled elastic tube- I: The linear approximation for long waves. Philosophical Magazine, 46, 199-221 (1955).

M. Zamir, The physics of pulsatile flow, Springer-Verlag, New York, (2000). 